\title{
Corticosteroids in acute respiratory distress syndrome: outcomes obscured by mortality
}

\author{
Dong Kyu Oh
}

Department of Pulmonary and Critical Care Medicine, Asan Medical Center, University of Ulsan College of Medicine, Seoul, Korea

Received: November 26, 2020 Accepted: December 6, 2020

\author{
Correspondence to \\ Dong Kyu Oh, M.D. \\ Department of Pulmonary and \\ Critical Care Medicine, Asan \\ Medical Center, University of \\ Ulsan College of Medicine, 88 \\ Olympic-ro 43-gil, Songpa-gu, \\ Seoul 05505, Korea \\ Tel: +82-2-3010-1861 \\ Fax: +82-2-3010-6968 \\ E-mail: synthesis83@hanmail.net \\ https://orcid.org/0000-0002- \\ 7511-9634
}

\section{See Article on Page 145-153}

The potential benefit and harm of using corticosteroids to manage acute respiratory distress syndrome (ARDS) have been evaluated since the 1980s [18]. However, studies have yielded contradictory results, and clinicians are confused whether corticosteroids are their friend or foe in the management of ARDS.

In an article published in The Korean Journal of Internal Medicine, Baek et al. [9] report the results of a propensitymatched cohort study that investigated the effect of corticosteroids administered in the early phase of ARDS. In that study, the use of corticosteroids was not associated with 28 days (odds ratio [OR], 1.031; 95\% confidence interval [CI], 0.657 to $1.618 ; p=0.895)$ and 90 days $(\mathrm{OR}$, 1.435; $95 \%$ CI, 0.877 to $2.348 ; p=0.151$ ) mortality rates after adjusting propensity scores. However, the corticosteroid-treated group showed a prolonged mechanical ventilation period (12 days in the corticosteroid-treated group vs. 8 days in the control group, $p<0.001$ ), which may have contributed to a higher incidence of pneumothorax (10.9\% vs. $3.7 \%, p=0.007$ ) and a longer stay in the intensive care unit ( 16 days vs. 10 days, $p<0.001$ ). Furthermore, bacteremia was significantly more frequent in the corticosteroid-treated group (41.1\% vs. $30.4 \%, p=0.019)$, suggesting harmful immunosuppression.

Corticosteroids reduce inflammation and fibrosis, which can be useful in the treatment of ARDS [1-8]. However, corticosteroid therapy has been reported to be ineffective or even harmful in the late phase of the disease [5]. In the Late Steroid Rescue Study (LaSRS), corticosteroids did not benefit the 60 days (29.2\% in the corticosteroid-treated group vs. $28.6 \%$ in the control group, $p=1.0$ ) or 180 days $(31.5 \%$ in the corticosteroid-treated group vs. $31.9 \%$ in the control group, $p=1.0$ ) mortality rate. Furthermore, the use of corticosteroids was associated with increased mortality rates $(35 \%$ vs. $8 \%, p=0.02$ for 60 days mortality; $44 \%$ vs. $12 \%, p=0.01$ for 180 days mortality) if administered $>14$ days after the onset of ARDS.

By contrast, in the early phase of ARDS, particularly before fibrosis develops, the effect of corticosteroids on mortality is contentious $[1-3,6-8]$. In the randomized controlled trial by Tongyoo et al. [6], administration of corticosteroids was not associated with 28 -day mortality (22.5\% vs. $27.3 \%$, relative risk, $0.82 ; 95 \% \mathrm{CI}, 0.50$ to $1.34 ; p=$ 0.51 ), in line with the findings of Baek et al. [9]. By contrast, a recent randomized controlled trial by Villar et al. [7] showed that treatment with corticosteroids was associated with decreased mortality at 
day 60 (21\% vs. 36\%; between group difference, $-15.3 \%$; 95\% CI, -25.9 to $-4.9 ; p=0.0047)$. The Randomized Evaluation of COVID-19 Therapy (RECOVERY) trial, which included patients with coronavirus disease 2019 (COVID19), also suggested a potential benefit of corticosteroids on 28-day mortality (age-adjusted rate ratio, $0.83 ; 95 \% \mathrm{CI}, 0.75$ to $0.93 ; p<0.001$ ) [8], indicating that there may be a subgroup that responds to the treatment.

Although mortality is an important endpoint, it is affected by numerous confounders, and thus tends to be poorly replicable in critically ill patients [10-12]. In such cases, secondary outcomes with softer endpoints provide considerable insight for clinicians. Therefore, the increased incidence of pneumothorax and bacteremia in the corticosteroidtreated group reported by Baek et al. [9] is remarkable. Other potential harmful effects of corticosteroids, such as neuromuscular weakness, immunosuppression, superimposed infection, and an increased blood glucose level, have been investigated [13]. The mineralocorticoid effect of steroids is also associated with adverse outcomes in patients with lung injury because it facilitates fluid and sodium retention [14].

Despite several limitations, the present study highlights the potential harm of corticosteroids, which is usually concealed by mortality. On the principle of primum non nocere (first, do no harm), there is insufficient evidence to support the routine use of corticosteroids in patients with ARDS. The administration of corticosteroids should be limited to subgroups that may respond to treatment (i.e., patients with COVID19-associated ARDS), and even in such cases clinicians should be aware of the potential harm of the treatment and closely monitor the patient for adverse events.

\section{Conflict of interest}

No potential conflict of interest relevant to this article was reported

\section{REFERENCES}

1. Bernard GR, Luce JM, Sprung CL, et al. High-dose corticosteroids in patients with the adult respiratory distress syndrome. N Engl J Med 1987;317:1565-1570.

2. Annane D, Sebille V, Bellissant E; Ger-Inf-o5 Study Group.
Effect of low doses of corticosteroids in septic shock patients with or without early acute respiratory distress syndrome. Crit Care Med 2006;34:22-30.

3. Meduri GU, Golden E, Freire AX, et al. Methylprednisolone infusion in early severe ARDS: results of a randomized controlled trial. Chest 2007;131:954-963.

4. Meduri GU, Headley AS, Golden E, et al. Effect of prolonged methylprednisolone therapy in unresolving acute respiratory distress syndrome: a randomized controlled trial. JAMA 1998;280:159-165.

5. Steinberg KP, Hudson LD, Goodman RB, et al. Efficacy and safety of corticosteroids for persistent acute respiratory distress syndrome. N Engl J Med 2006;354:1671-1684.

6. Tongyoo S, Permpikul C, Mongkolpun W, et al. Hydrocortisone treatment in early sepsis-associated acute respiratory distress syndrome: results of a randomized controlled trial. Crit Care 2016;20:329.

7. Villar J, Ferrando C, Martinez D, et al. Dexamethasone treatment for the acute respiratory distress syndrome: a multicentre, randomized controlled trial. Lancet Respir Med 2020;8:267-276.

8. RECOVERY Collaborative Group, Horby P, Lim WS, et al. Dexamethasone in hospitalized patients with Covid-19: preliminary report. N Engl J Med 2020 Jul 17 [Epub]. https://doi.org/10.1056/NEJMoa2021436.

9. Baek MS, Lee Y, Hong SB, Lim CM, Koh Y, Huh JW. Effect of corticosteroid therapy in the early phase of acute respiratory distress syndrome: a propensity-matched cohort study. Korean J Intern Med 2021;36:145-153.

10. Petros AJ, Marshall JC, van Saene HK. Should morbidity replace mortality as an endpoint for clinical trials in intensive care? Lancet 1995;345:369-371.

11. Ospina-Tascon GA, Buchele GL, Vincent JL. Multicenter, randomized, controlled trials evaluating mortality in intensive care: doomed to fail? Crit Care Med 2008;36:13111322.

12. Kress JP. Mortality is the only relevant outcome in ARDS: no. Intensive Care Med 2015;41:144-146.

13. Cronin L, Cook DJ, Carlet J, et al. Corticosteroid treatment for sepsis: a critical appraisal and meta-analysis of the literature. Crit Care Med 1995;23:1430-1439.

14. Zuckerman S, Palmer A, Hanson DA. The effect of steroid hormones on the water content of tissues. J Endocrinol 1950;6:261-276. 